\title{
Subcarrier and Power Allocation for Multiuser MI MO-OFDM Systems with Various Detectors
}

\author{
Jing Mao ${ }^{1}$, Chen $\mathrm{Chen}^{1}$, Lin Bai ${ }^{2}$, Haige Xiang ${ }^{1}$ and Jinho $\mathrm{Choi}^{3}$ \\ ${ }^{1}$ State Key Laboratory of Advanced Optical Communication Systems and Networks, Peking University \\ Beijing, China \\ [e-mail: \{maojing, c.chen, Xianghg\}@pku.edu.cn] \\ ${ }^{2}$ School of Electronic and Information Engineering, Beihang University \\ Beijing, China \\ [e-mail: l.bai@buaa.edu.cn] \\ ${ }^{3}$ School of Information and Communications, Gwangju Institute of Science and Technology (GIST) \\ Korea \\ [e-mail: jchoi0114@gist.ac.kr]
}

Received February 17, 2017; revised May 28, 2017; accepted June 22, 2017;

published October 31, 2017

\begin{abstract}
Resource allocation plays a crucial role in multiuser multiple input multiple output orthogonal frequency division multiplexing (MIMO-OFDM) systems to improve overall system performance. While previously proposed resource allocation algorithms are mainly designed from the point of view of the information-theoretic, we formulate the resource allocation problem as an average bit error rate (BER) minimization problem subject to a total power constraint when considering employing realistic MIMO detection techniques. Subsequently, we derive the optimal subcarrier and power allocation algorithms for three types of well-known MIMO detectors, including the maximum likelihood (ML) detector, linear detectors, and successive interference cancellation (SIC) detectors. To reduce the complexity, we also propose a two-step suboptimal algorithm that separates subcarrier and power allocation for each detector. We also analyze the diversity gain of the proposed suboptimal algorithms for various MIMO detectors. Simulation results confirm that the proposed suboptimal algorithm for each detector can achieve a comparable performance with the optimal allocation with a much lower complexity. Moreover, it is shown that the suboptimal algorithms perform better than the conventional algorithms that are known in the literature.
\end{abstract}

Keywords: multiuser, MIMO-OFDM, subcarrier and power allocation, MIMO detector, BER

A preliminary version of this paper appeared in IEEE VTC 2016-Spring, May 15-18, Nanjing, China. This version includes a concrete derivation of the proposed algorithms and diversity analysis of the suboptimal algorithms for various detectors. This research was supported in part by the National Science Foundation of China (NSFC, Grant No.61471008). 


\section{Introduction}

Multiple input multiple output (MIMO) technology can achieve a higher spectral gain by transmitting independent data streams over a group of antennas. In the meantime, orthogonal frequency division multiplexing (OFDM) has been used to mitigate the inter-symbol interference (ISI) in wireless broadband systems. The combination of these two technologies, which is called MIMO-OFDM, has been adopted as the technology for several broadband wireless standards, including the IEEE 802.11ac for wireless local networking [1], IEEE 802.16e for metropolitan area networks [2], and third generation partnership project (3GPP) long term evolution (LTE) for cellular networks. Moreover, MIMO-OFDM technology plays a vital role in the fourth-generation wireless communication systems.

It is well known that by dynamically allocating resources among users in the multiuser systems, the multiuser diversity can be exploited to enhance power or spectrum efficiency or equivalently, improve the systems' bit error rate (BER) performance [3][4]. In most of existing literature on adaptive resource allocation (subcarrier, power, bit, antenna, etc.) in multiuser MIMO-OFDM systems, the proposed algorithms are mainly designed to maximize the data rate/capacity subject to a power constraint [5][6][7][8] or to minimize the overall transmit power subject to a rate constraint [9][10][11][12]. In [5], a low-complexity proportional rate-adaptive radio resource (subcarrier and power) allocation algorithm is proposed to maximize the sum-rate capacity of the system under a total power constraint. In [9], a subcarrier and bit allocation scheme is obtained with a goal of minimizing the overall transmit power while meeting a constant bit-rate.

However, the works mentioned above design the resource allocation algorithm from the point of the view of the information-theoretic without considering the constraint of the MIMO detector. In a practical transmission, receivers at users may not be ideal and their performance strongly depends on detectors that are actually employed. When a realistic MIMO detection technique is employed, the BER can be considered as a performance criterion with its direct relation to the performance of the actual detector employed. Clearly, resource allocation in sense of maximizing rate/capacity cannot be directly applied to reduce BER. Thus, there have also been some researchers on the BER minimization [13][14][15][16]. In [13], a BER-optimized power allocation algorithm is investigated in the point-to-point OFDM system. For the MIMO system, Wang et al. take the BER as the optimization criterion and propose a unified power allocation for a variety of detection method [14], including zero forcing (ZF), successive interference cancellation (SIC), and ordered SIC (OSIC). However, to the best of our knowledge, the BER-optimized resource allocation problem for MIMO-OFDM systems has not been studied yet in the literature.

In this paper, we investigate the subcarrier and power allocation towards BER minimization for multiuser MIMO-OFDM systems when realistic MIMO detection methods are employed. The main contributions of this paper are summarized as follows:

1) The BER optimization resource allocation problem for multiuser MIMO-OFDM systems is formulated. As the BER is depending on the realistic MIMO detection techniques, we solve the problem for three types of well-known MIMO detectors.

2) To reduce the complexity, a suboptimal subcarrier and closed-form power allocation algorithm is proposed for each detector. We also analyze the diversity gain of the proposed suboptimal algorithms for various MIMO detectors. 
3) It is shown that, for each detector, the proposed suboptimal resource allocation algorithms can achieve a close performance to the optimal algorithm and perform better than the conventional algorithms.

This paper is organized as follows. Section 2 introduces the system model and formulates the problem of BER minimization for the given multiuser MIMO-OFDM system. In Section 3, the optimal and suboptimal subcarrier and power allocation algorithms for three types of detectors are proposed. Section 4 presents the complexity and diversity analysis. Simulation results are presented in Section 5, followed by the concluding remarks in Section 6 .

Notations: Vectors and matrices are denoted by lowercase and uppercase boldface letters, respectively. $(\cdot)^{\mathrm{T}}$ and $(\cdot)^{\mathrm{H}}$ denote the transpose and conjugate transpose operation, respectively. $(\cdot)^{\dagger}$ denotes the matrix Moore-Penrose pseudoinverse and $\|\cdot\|$ denotes the vector Euclidean norm. $\mathbf{I}_{N}$ denotes the $N \times N$ identity matrix and $\mathbf{e}_{i}^{N}$ stands for the $i$-th column of the $\mathbf{I}_{N} \cdot[\mathbf{X}]_{i, j}$ denotes the $(i, j)$-th element of the matrix $\mathbf{X} . \mathbb{E}[\cdot]$ denotes statistical expectation. $\mathbf{n} \sim \mathcal{C N}(\mathbf{m}, \mathbf{R})$ denotes a circularly symmetric complex Gaussian (CSCG) vector $\mathbf{n}$ with mean $\mathbf{m}$ and covariance matrix $\mathbf{R}$.

\section{System Model and Problem Formulation}

Consider a multiuser MIMO-OFDM system with a base station (BS) that communications with $K$ user terminals via $N$ subcarriers in downlink channels. Each user is equipped with $Q$ receive antennas and the $\mathrm{BS}$ is equipped with $M$ transmit antennas, where $Q \geq M$. Fig. 1 depicts the structure of a downlink multiuser MIMO-OFDM system. For simplicity, the block fading model [17] is considered, i.e., the channel is invariant during each transmission block. Furthermore, the cyclic prefix is assumed to be sufficient long to avoid inter-block interference caused by the multipath propagation. The MIMO frequency-domain channel on subcarrier $n$ between the base station and user $k$ can be characterized by a $Q \times M$ matrix $\mathbf{H}_{k, n}$, where $k \in \mathcal{K}=\{1,2, \ldots, K\}$ and $n \in \mathcal{N}=\{1,2, \ldots, N\}$. Assuming that the time domain channel responses have $L$ delayed multipath taps, and the $k$-th user's channel matrix for the $l$-th tap is denoted by $\mathbf{G}_{k}(l), l \in \mathcal{L}=\{1,2, \ldots, L\}, \mathbf{H}_{k, n}$ can be expressed as

$$
\mathbf{H}_{k, n}=\frac{1}{\sqrt{N}} \sum_{l=1}^{L} \mathbf{G}_{k}(l) \exp \left(\frac{-j 2 \pi(n-1)(l-1)}{N}\right) .
$$

In this paper, we assume that the channel state information (CSI) is perfectly known by the BS. At each transmission block, the BS performs the subcarrier and power allocation for the downlink. Then, the result of the subcarrier and power allocation is sent to all users via a separate control channel. The data from the BS is transmitted using the spatial multiplexing technique. Thus, the transmitted signal can be expressed as $\mathbf{x}_{k, n}=\left(\mathbf{x}_{k, n}(1), \ldots, \mathbf{x}_{k, n}(M)\right)^{\mathrm{T}}$, where $\mathbf{x}_{k, n}(m)$ represents the transmitted signal for user $k$ on subcarrier $n$ from transmit antenna $m$. The transmitted signals are assumed to be independent vectors with zero means and covariance matrices $\mathbf{I}_{M}=\mathbb{E}\left[\mathbf{x}_{k, n} \mathbf{x}_{k, n}^{\dagger}\right]$.

When multiple users share a certain subcarrier, the transmission systems that include linear pre- and post-processing can be considered to spatially separate the users [18]. For simplicity, 


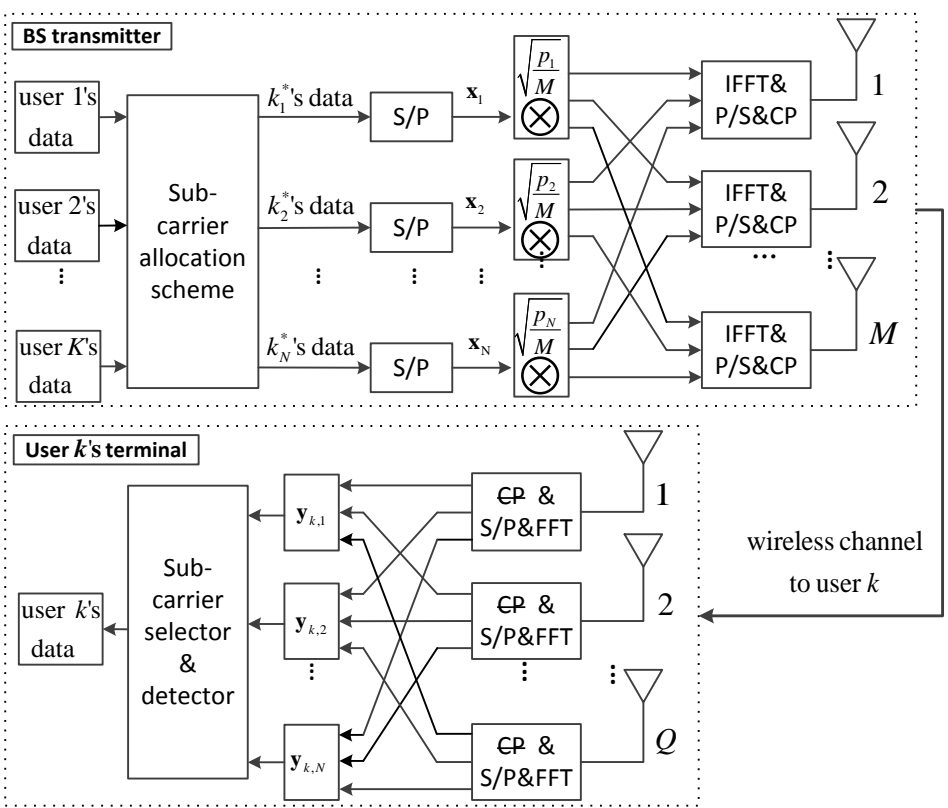

Fig. 1. Structure of the downlink multiuser MIMO-OFDM system

we do not allow more than one user to share a subcarrier. The allocation of the $k$-th user to subcarrier $n$ is indicated by $\rho_{k, n}$. That is, if the $k$-th user is allocated to subcarrier $n$, $\rho_{k, n}=1$; otherwise, $\rho_{k, n}=0$. With the subcarrier allocation $\left\{\rho_{k, n}\right\}$ is known, the user $k_{n}^{*}$, which subcarrier $n$ is allocated to, is determined. Thus, the transmitted signal on subcarrier $n$ is denoted by $\mathbf{x}_{n}$ as the user index $k$ can be omitted. Denote by $p_{n}$ the transmit power of subcarrier $n$, and assume the power is equally allocated on each antenna, then the baseband input-output relationship is represented as

$$
\mathbf{y}_{k, n}=\mathbf{H}_{k, n} \sqrt{\frac{p_{n}}{M}} \mathbf{x}_{n}+\mathbf{v}_{k, n}, \forall k, \forall n,
$$

where $\mathbf{y}_{k, n}$ is the $Q \times 1$ receive signal vector for user $k$ on the $n$-th subcarrier and $\mathbf{v}_{k, n} \sim \mathcal{C N}\left(\mathbf{0}, \sigma^{2} \mathbf{I}_{Q}\right)$ is the noise vector. It is obvious that the signal received on subcarrier $n$ is only detected by the user $k_{n}^{*}$ with the subcarrier selector.

Our objective is to find the optimal subcarrier allocation $\left\{\rho_{k, n}\right\}$ and the power allocation $\left\{p_{n}\right\}$ that minimize the overall average BER subject to a total power constraint. The optimization problem is given as follows:

$$
\begin{aligned}
\min _{\left\{\rho_{k, n}\right\},\left\{p_{n}\right\}} & \frac{1}{N} \sum_{k=1}^{K} \sum_{n=1}^{N} \rho_{k, n} B E R_{k, n} \\
\text { s.t. } & \sum_{\mathrm{n}=1}^{\mathrm{N}} p_{n}=P_{\mathrm{T}} \\
& \sum_{k=1}^{K} \rho_{k, n}=1, \forall n,
\end{aligned}
$$


where $B E R_{k, n}$ and $P_{T}$ represent the BER for user $k$ on the subcarrier $n$ and the total power, respectively.

\section{Subcarrier and Power Allocation for Various Detectors}

In this section, we investigate the subcarrier and power allocation for three types of well-known detectors, including the maximum likelihood (ML) detector, linear detectors and SIC detectors. For each type of detector, we first obtain the optimal resource allocation algorithm via the method of Lagrange multipliers. A suboptimal resource allocation algorithm is also proposed to reduce the computational complexity. For simplicity, only binary phase shift keying (BPSK) is considered in this paper. If a higher-order modulation is employed, symbol error rate (SER) or word error rate (WER) can be considered.

\subsection{Detector}

It is noted that the optimal performance can be achieved by the ML detector where the estimate of the transmitted signal is given by $\tilde{\mathbf{x}}_{n}=\arg \min _{\mathbf{x} \in \mathcal{X}}\left\|\mathbf{y}_{k, n}-\mathbf{H}_{k, n} \sqrt{p_{n} / M} \mathbf{x}\right\|^{2}$, where $\mathcal{X}$ is a set of the candidate vector signals. A pairwise error probability (PEP) can be derived as in [19],

$$
P_{k, n}\left(\mathbf{x}_{n} \rightarrow \tilde{\mathbf{x}}_{n}\right)=Q\left(\sqrt{\frac{p_{n}\left\|\mathbf{H}_{k, n} \mathbf{d}_{n}\right\|^{2}}{2 M \sigma^{2}}}\right),
$$

where $Q(x)=1 / \sqrt{2 \pi} \int_{x}^{\infty} e^{-t^{2} / 2} d t$ denotes the Gaussian-Q function and $\mathbf{d}_{n}=\mathbf{x}_{n}-\tilde{\mathbf{x}}_{n}$ is called difference vector. Here, $P_{k, n}\left(\mathbf{x}_{n} \rightarrow \tilde{\mathbf{x}}_{n}\right)$ is the PEP of confusing $\mathbf{x}_{n}$ with $\tilde{\mathbf{x}}_{n}$ when $\mathbf{x}_{n}$ is transmitted for user $k$ on subcarrier $n$. As obtaining an explicit expression for the exact BER of the ML detector for the MIMO system is well known to be difficult, we replace the $B E R_{k, n}$ in Problem (3) with an upper bound which can be expressed as

$$
\sum_{\mathbf{x}_{n} \in \mathcal{X}} \frac{1}{2^{M}} \sum_{\substack{\tilde{x}_{n} \in \mathcal{X} \\ \tilde{\mathbf{x}}_{n} \neq \mathbf{x}_{n}}} \frac{N_{e}\left(\mathbf{x}_{n} \rightarrow \tilde{\mathbf{x}}_{n}\right)}{M} P_{k, n}\left(\mathbf{x}_{n} \rightarrow \tilde{\mathbf{x}}_{n}\right),
$$

where $N_{e}\left(\mathbf{x}_{n} \rightarrow \tilde{\mathbf{x}}_{n}\right)$ denotes the number of the error bits when the detected signal is not $\mathbf{x}_{n}$ but $\tilde{\mathbf{x}}_{n}$.

\subsubsection{Optimal resource allocation algorithm}

Clearly, (3) is an NP-hard problem and an exhaustive searching algorithm can be used. We can enumerate all possible of subcarrier allocation $\left\{\rho_{k, n}\right\}$. With the subcarrier allocation is known (i.e., the $k_{n}^{*}$ 's are given), the remaining problem to allocate power can be written as

$$
\begin{aligned}
& \min _{\left\{p_{n}\right\}} \frac{1}{N} \sum_{n=1}^{N} B E R_{k_{n}^{*}, n} \\
& \text { s.t. } \sum_{n=1}^{N} p_{n}=P_{T} .
\end{aligned}
$$


As Problem (6) is a convex optimization problem, we can solve it by the method of Lagrange multipliers with the following unconstrained optimization problem:

$$
L=\frac{1}{N} \sum_{n=1}^{N} B E R_{k_{n}^{*}, n}+\theta\left(\sum_{n=1}^{N} p_{n}-P_{T}\right),
$$

where $\theta$ is the Lagrangian multiplier. Let the derivation of (7) with respect to $p_{n}$ be zero, and we have

$$
\frac{\partial L}{\partial p_{n}}=\frac{1}{N} \frac{\partial B E R_{k_{n}^{*}, n}}{\partial p_{n}}+\theta=0, \forall n .
$$

The derivation of $B E R_{k_{n}^{*}, n}$ with respect to $p_{n}$ is given by

$$
\begin{aligned}
\frac{\partial B E R_{k_{n}^{*}, n}}{\partial p_{n}} & =\frac{-1}{4 M \sqrt{M \pi} 2^{M} \sigma} \sum_{\mathbf{x}_{n} \in \mathcal{X}} \sum_{\substack{\tilde{x}_{n} \in \mathcal{X} \\
\tilde{\mathbf{x}}_{n} \neq \mathbf{x}_{n}}} N_{e}\left(\mathbf{x}_{n} \rightarrow \tilde{\mathbf{x}}_{n}\right) \\
& \times \exp \left(-\frac{p_{n}\left\|\mathbf{H}_{k_{n}^{*}, n} \mathbf{d}_{n}\right\|^{2}}{4 M \sigma^{2}}\right) \sqrt{\frac{\left\|\mathbf{H}_{k_{n}^{*}, n} \mathbf{d}_{n}\right\|^{2}}{p_{n}}} .
\end{aligned}
$$

From (8) and (9), we have the following result

$$
\sum_{\substack { \mathbf{x}_{n} \in \mathcal{X} \\
\begin{subarray}{c}{\tilde{\mathbf{x}}_{n} \in \mathcal{X} \\
\tilde{\mathbf{x}}_{n} \neq \mathbf{x}_{n}{ \mathbf { x } _ { n } \in \mathcal { X } \\
\begin{subarray} { c } { \tilde { \mathbf { x } } _ { n } \in \mathcal { X } \\
\tilde { \mathbf { x } } _ { n } \neq \mathbf { x } _ { n } } }\end{subarray}} N_{e}\left(\mathbf{x}_{n} \rightarrow \tilde{\mathbf{x}}_{n}\right) \times \exp \left(-\frac{p_{n}\left\|\mathbf{H}_{k_{n}^{*}, n} \mathbf{d}_{n}\right\|^{2}}{4 M \sigma^{2}}\right) \sqrt{\frac{\left\|\mathbf{H}_{k_{n}^{*}, n} \mathbf{d}_{n}\right\|^{2}}{p_{n}}}=\tilde{\theta}, \forall n,
$$

where $\tilde{\theta}=4 N M \sqrt{M \pi} 2^{M} \sigma \theta$ is chosen to satisfy the power constraint. Since the left-hand side of (10) is a continuous and strictly decreasing function of $p_{n}$, the solution is unique due to one-to-one relation between $p_{n}$ and $\tilde{\theta}$. Obviously, it is not a close-form power allocation, and the solution of (10) must be obtained numerically.

As mentioned above, solving the optimal power allocation problem needs a numerical search, and the computational complexity for the subcarrier allocation is $\mathcal{O}\left(K^{N}\right)$. As a consequence, it is necessary to propose a suboptimal algorithm of lower complexity.

\subsubsection{Suboptimal resource allocation algorithm}

We focus on a low-complexity and suboptimal solution of (3) for the ML detector here. The PEP has the following upper bound:

$$
Q\left(\sqrt{\frac{p_{n}\left\|\mathbf{H}_{k, n} \mathbf{d}_{n}\right\|^{2}}{2 M \sigma^{2}}}\right) \leq \max _{\mathbf{d} \in \mathcal{D}} Q\left(\sqrt{\frac{p_{n}\left\|\mathbf{H}_{k, n} \mathbf{d}\right\|^{2}}{2 M \sigma^{2}}}\right),
$$

where $\mathcal{D}=\left\{\mathbf{d}=\mathbf{x}-\tilde{\mathbf{x}}_{n} \mid \mathbf{x}, \tilde{\mathbf{x}}_{n} \in \mathcal{X}, \mathbf{x} \neq \tilde{\mathbf{x}}_{n}\right\}$. Then, (5) can be upper bounded as 


$$
\begin{aligned}
B E R_{k, n} & \leq \max _{\mathbf{d} \in \mathcal{D}} Q\left(\sqrt{\frac{p_{n}\left\|\mathbf{H}_{k, n} \mathbf{d}\right\|^{2}}{2 M \sigma^{2}}}\right) \times \sum_{\mathbf{x}_{n} \in \mathcal{X}} \frac{1}{2^{M}} \sum_{\substack{\tilde{x}_{n} \in \mathcal{X} \\
\tilde{\mathbf{x}}_{n} \neq \mathbf{x}_{n}}} \frac{N_{e}\left(\mathbf{x}_{n} \rightarrow \tilde{\mathbf{x}}_{n}\right)}{M} \\
& \leq\left(2^{M}-1\right) Q\left(\sqrt{\frac{p_{n} \min _{\mathbf{d} \in \mathcal{D}}\left\|\mathbf{H}_{k, n} \mathbf{d}\right\|^{2}}{2 M \sigma^{2}}}\right) .
\end{aligned}
$$

To minimize the upper bound (12) , the subcarrier allocation scheme becomes

where [20]

$$
\rho_{k, n}=\left\{\begin{array}{l}
1, \quad \text { if } k=k_{n}^{*}, \\
0, \quad \text { otherwise, }
\end{array} \forall k, \forall n,\right.
$$

$$
k_{n}^{*}=\arg \max _{k}\left\{\min _{\mathbf{d} \in \mathcal{D}}\left\|\mathbf{H}_{k, n} \mathbf{d}\right\|\right\} .
$$

Once the subcarrier allocation $\left\{\rho_{k, n}\right\}$ is known, we could allocate the power via the optimal power allocation algorithm as stated above. However, its complexity to find the optimal solution can be still high as the parameter $\tilde{\theta}$ must be numerically obtained. Replacing the $B E R_{k, n}$ with the upper bound in (12), we can use a similar algorithm in [14][21] which allocates more power to the best subcarriers when average signal noise ratio (SNR) is low and allocates more power to the worst subcarriers average SNR is high. The derivation of the algorithm can be found in Appendix, and the power allocation for the ML detector is given as follows:

$$
p_{n}=\frac{P_{T} * \zeta_{n}}{1+\zeta_{n}^{2}}\left(\sum_{j=1}^{N} \frac{\zeta_{j}}{1+\zeta_{j}^{2}}\right)^{-1}, \forall n
$$

where $\zeta_{n}=P_{T} /\left(2 M \sigma^{2}\right) \min _{\mathbf{d} \in \mathcal{D}}\left\|\mathbf{H}_{k_{n}^{*}, n} \mathbf{d}\right\|^{2}$.

\subsection{Linear Detectors}

With a linear detector, the estimate of the transmitted signal $\mathbf{x}_{n}$ is given by $\tilde{\mathbf{x}}_{n}=\mathbf{G y}_{k, n}$, where $\mathbf{G}$ is a linear filter. Then, the post-processing SNR of the signal transmitted on the antenna $m$ is given by

$$
S N R_{k, n, m}=\frac{p_{n}\left|\mathbf{g}_{m}^{\prime} \mathbf{h}_{k, n, m}\right|^{2}}{M \sigma^{2}\left\|\mathbf{g}_{m}^{\prime}\right\|^{2}+p_{n} \sum_{j \neq m}\left|\mathbf{g}_{m}^{\prime} \mathbf{h}_{k, n, j}\right|^{2}},
$$

where $\mathbf{g}_{i}^{\prime}$ is the $i$-th row of $\mathbf{G}$ and $\mathbf{h}_{k, n, i}$ is the $i$-th column of $\mathbf{H}_{k, n}$. For the ZF detector, we have $\mathbf{G}=\mathbf{H}_{k, n}^{\dagger}$. Then, (16) is simplified to

$$
S N R_{k, n, m}^{\mathrm{ZF}}=\frac{p_{n}}{M \sigma^{2}\left[\left(\mathbf{H}_{k, n}^{\mathrm{H}} \mathbf{H}_{k, n}\right)^{-1}\right]_{m, m}} \stackrel{\text { def }}{=} \frac{p_{n} \gamma_{k, n, m}}{M \sigma^{2}},
$$

where $1 /\left[\left(\mathbf{H}_{k, n}^{\mathrm{H}} \mathbf{H}_{k, n}\right)^{-1}\right]_{m, m}$ is denoted by $\gamma_{k, n, m}$. Thus, the average BER of the $k$-th user on 
subcarrier $n$ can be found as [19]

$$
B E R_{k, n}=\frac{1}{M} \sum_{m=1}^{M} Q\left(\sqrt{\frac{2 p_{n} \gamma_{k, n, m}}{M \sigma^{2}}}\right) .
$$

For the minimum mean-square error (MMSE) detector, we have $\mathbf{G}=\left[\mathbf{H}_{k, n}^{\mathrm{H}} \mathbf{H}_{k, n}+M \sigma^{2} / p_{n} \mathbf{I}_{M}\right]^{-1} \mathbf{H}_{k, n}^{\mathrm{H}}$. Then, (16) is rewritten as

$$
S N R_{k, n, m}^{\mathrm{MMSE}}=\frac{p_{n}}{M \sigma^{2}\left[\left(\mathbf{H}_{k, n}^{\mathrm{H}} \mathbf{H}_{k, n}+M \sigma^{2} / p_{n} \mathbf{I}_{M}\right)^{-1}\right]_{m, m}}-1 .
$$

The analysis of BER is not straightforward for the MMSE detector as the decision statistic is not Gaussian but the sum of a Gaussian random variable and a binomial random variable. For BPSK, Verdú [22] provides the exact BER formula which is complex with requiring computing $2^{M-1} \mathrm{Q}$-functions. Nevertheless, the MMSE detector becomes the ZF detector as the SNR increases. Therefore, in this section we only consider the ZF detector.

\subsubsection{Optimal resource allocation algorithm}

We still use a full search algorithm to obtain the optimal solution to Problem (3). With the given $\left\{\rho_{k, n}\right\}$ (i.e., $k_{n}^{*}$ 's are determined), the optimal solution to the power allocation problem (6) can be found by using the standard Lagrange multiplier technique. The Lagrangian of this problem is

$$
L=\frac{1}{N} \sum_{n=1}^{N} \frac{1}{M} \sum_{m=1}^{M} Q\left(\sqrt{\frac{2 p_{n} \gamma_{k_{n}^{*}, n, m}}{M \sigma^{2}}}\right)+\mu\left(\sum_{n=1}^{N} p_{n}-P_{T}\right),
$$

where $\mu$ is the Lagrange multiplier. Then, the solution can be obtained by solving $\partial L / \partial p_{n}=0$. Thus, we obtain the following power allocation:

$$
\sum_{m=1}^{M} \sqrt{\frac{\gamma_{k_{n}^{*}, n, m}}{M \sigma^{2} p_{n}}} \times \exp \left(-\frac{p_{n} \gamma_{k_{n}^{*}, n, m}}{M \sigma^{2}}\right)=\bar{\mu},
$$

where $\bar{\mu}=2 \mu M N \sqrt{\pi}$ is chosen to satisfy the total power constraint. A numerical solution can be obtained by the same method used for the ML detector.

There are $K^{N}$ combinations for the subcarrier allocation, and the solution to (19) should be numerically obtained for each combination of subcarrier allocation. To reduce the computational complexity, we can reduce the number of combinations for the subcarrier allocation as follows. At the beginning, define the set of the users might use the subcarrier $n$ as $\mathcal{K}_{n}$.

1) Initialization set $\mathcal{K}_{n}=\mathcal{K}$, for all $n \in \mathcal{N}$.

2) Iteration

for each $n \in \mathcal{N}$

a) calculate the $\gamma_{k, n, m}$, for all $k \in \mathcal{K}$, for all $m=1,2, \cdots, M$.

b) for $k=1: K$

$$
\begin{aligned}
& \text { sort } \gamma_{k, n, m} \text { in ascending or descending order, i.e., } \gamma_{k, n, m_{i}} \leq \gamma_{k, n, m_{i+1}} \text { or } \\
& \gamma_{k, n, m_{i}} \geq \gamma_{k, n, m_{i+1}}, i=1, \ldots, M-1
\end{aligned}
$$


end for

c) for $k_{1}=1: K-1$

$$
\begin{aligned}
& \text { for } k_{2}=k_{1}+1: K \\
& \text { if } \gamma_{k_{1}, n, m_{i}} \leq \gamma_{k_{2}, n, m_{i}} \text { for all } i=1,2, \cdots, M \\
& \qquad \mathcal{K}_{n}=\mathcal{K}_{n}-\left\{k_{1}\right\} \\
& \text { else if } \gamma_{k_{1}, n, m_{i}} \geq \gamma_{k_{2}, n, m_{i}} \text { for all } i=1,2, \cdots, M \\
& \qquad \mathcal{K}_{n}=\mathcal{K}_{n}-\left\{k_{2}\right\} \\
& \text { end if }
\end{aligned}
$$

end for.

As a result, the number of the combinations for the subcarrier allocation becomes $\prod_{n=1}^{N} \operatorname{Card}\left(\mathcal{K}_{n}\right)$, where $\operatorname{Card}(\cdot)$ denotes the cardinality of a set. Although we reduce the number of combinations of subcarrier allocation, the complexity of the optimal algorithm is still prohibitively high. To further reduce the complexity, we propose a simple suboptimal algorithm in the next section.

\subsubsection{Suboptimal resource allocation algorithm}

As shown in Section 3.1.2 when considering the ML detector, subcarrier $n$ should be assigned to the user with the minimum $B E R_{k, n}$. Based on the Rayleigh-Ritz Theorem [23], we can get

$$
\begin{gathered}
{\left[\left(\mathbf{H}_{k, n}^{\mathrm{H}} \mathbf{H}_{k, n}\right)^{-1}\right]_{m, m}=\frac{\left(\mathbf{e}_{m}^{M}\right)^{\mathrm{H}}\left[\left(\mathbf{H}_{k, n}^{\mathrm{H}} \mathbf{H}_{k, n}\right)^{-1}\right] \mathbf{e}_{m}^{M}}{\left(\mathbf{e}_{m}^{M}\right)^{\mathrm{H}} \mathbf{e}_{m}^{M}}} \\
\quad \leq \lambda_{\text {max }}\left[\left(\mathbf{H}_{k, n}^{\mathrm{H}} \mathbf{H}_{k, n}\right)^{-1}\right]=\left[\lambda_{\text {min }}\left(\mathbf{H}_{k, n}^{\mathrm{H}} \mathbf{H}_{k, n}\right)\right]^{-1},
\end{gathered}
$$

where $\lambda_{\max }(\mathbf{A})$ and $\lambda_{\min }(\mathbf{A})$ denote the maximum and minimum eigenvalue of matrix $\mathbf{A}$, respectively. From (18) and (20), we can obtain the following upper bound:

$$
B E R_{k, n} \leq Q\left(\sqrt{\frac{2 p_{n} \lambda_{\text {min }}\left(\mathbf{H}_{k, n}^{\mathrm{H}} \mathbf{H}_{k, n}\right)}{M \sigma^{2}}}\right) .
$$

This shows that the subcarrier can be allocated to the user with the maximum of the minimum eigenvalues of $\mathbf{H}_{k, n}^{\mathrm{H}} \mathbf{H}_{k, n}$. In other words, for the $n$-th subcarrier, we have $\rho_{k_{n}^{*}, n}=1$, $\rho_{k^{\prime}, n}=0, \forall k^{\prime} \neq k_{n}^{*}$, where

$$
k_{n}^{*}=\arg \max _{k} \lambda_{\min }\left(\mathbf{H}_{k, n}^{\mathrm{H}} \mathbf{H}_{k, n}\right) .
$$

Then, we use the suboptimal power allocation algorithm as described in Appendix based on the upper bound in (21). Thus, $p_{n}$ becomes

$$
p_{n}=\frac{P_{T} * \zeta_{n}}{1+\zeta_{n}^{2}}\left(\sum_{j=1}^{N} \frac{\zeta_{j}}{1+\zeta_{j}^{2}}\right)^{-1}, \forall n,
$$


where $\zeta_{n}=2 P_{T} /\left(M \sigma^{2}\right) \lambda_{\min }\left(\mathbf{H}_{k_{n}^{*}, n}^{\mathrm{H}} \mathbf{H}_{k_{n}^{*}, n}\right)$.

Besides(21), we can also get another upper bound from (18) as follows:

$$
B E R_{k, n} \leq Q\left(\sqrt{\frac{2 p_{n} \min _{m} \gamma_{k, n, m}}{M \sigma^{2}}}\right)=Q\left(\sqrt{\frac{2 p_{n}}{M \sigma^{2} \max _{m}\left[\left(\mathbf{H}_{k, n}^{\mathrm{H}} \mathbf{H}_{k, n}\right)^{-1}\right]_{m, m}}}\right) .
$$

Based on the upper bound in (24), the user with the minimum of the maximum diagonal elements of $\left(\mathbf{H}_{k, n}^{\mathrm{H}} \mathbf{H}_{k, n}\right)^{-1}$ is assigned to subcarrier $n$ as follows:

$$
k_{n}^{*}=\arg \min _{k}\left\{\max _{m}\left[\left(\mathbf{H}_{k, n}^{\mathrm{H}} \mathbf{H}_{k, n}\right)^{-1}\right]_{m, m}\right\} .
$$

The power allocation is the same as that expressed in (23) where $\zeta_{n}=2 P_{T} /\left(M \sigma^{2}\right) \min _{m} \gamma_{k_{n}^{*}, n, m}$.

The above two algorithms will be referred to as the max-min eigenvalue (in short, M_eig) algorithm and the min-max diagonal element (in short, M_diag) algorithm, respectively.

\subsection{SIC Detectors}

There are a lot SIC detectors, including ZF-SIC, MMSE-SIC and ordered SIC detectors. When an MMSE decision feedback equalizer (DFE) is carried out at each stage, the exact BER formula is complex. However, as the MMSE-DFE becomes the ZF-DFE with a high SNR, we only derive the resource allocation algorithm for the ZF-SIC detector in this section. Moreover, the algorithm for the ZF-SIC detector can be easily applied to the ordered ZF-SIC detector.

The channel matrix $\mathbf{H}_{k, n}$ is QR factorized as $\mathbf{H}_{k, n}=\mathbf{Q}_{k, n} \mathbf{R}_{k, n}$, where $\mathbf{Q}_{k, n}$ is unitary and $\mathbf{R}_{k, n}$ is upper triangular. Premultiplying $\mathbf{Q}_{k, n}^{\mathrm{H}}$ to $\mathbf{y}_{k, n}$, we have

$$
\mathbf{Q}_{k, n}^{\mathrm{H}} \mathbf{y}_{k, n}=\mathbf{Q}_{k, n}^{\mathrm{H}}\left(\mathbf{H}_{k, n} \sqrt{\frac{p_{n}}{M}} \mathbf{x}_{n}+\mathbf{v}_{k, n}\right)=\mathbf{R}_{k, n} \sqrt{\frac{p_{n}}{M}} \mathbf{x}_{n}+\mathbf{u}_{k, n},
$$

where $\mathbf{u}_{k, n}$ has the same statistical properties of $\mathbf{v}_{k, n}$. It is a valid assumption that the error propagation can be ignored at moderate-to-high SNR, so the SNR of the $m$-th signal stream is

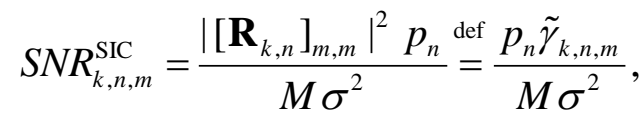

where $\tilde{\gamma}_{k, n, m}$ denotes $\left|\left[\mathbf{R}_{k, n}\right]_{m, m}\right|^{2}$. Note that (26) has the same form as the SNR for the ZF detector in (17). Thus, the same procedure is easily adopted to derive the optimal subcarrier and power allocation algorithm for the ZF-SIC detector. We also enumerate the subcarrier allocation to allocate the power for a given subcarrier allocation as follows:

$$
\sum_{m=1}^{M} \sqrt{\frac{\tilde{\gamma}_{k_{n}^{*}, n, m}}{M \sigma^{2} p_{n}}} \times \exp \left(-\frac{p_{n} \tilde{\gamma}_{k_{n}^{*}, n, m}}{M \sigma^{2}}\right)=\tilde{\mu},
$$

where $\tilde{\mu}$ is chosen to satisfy the total power constraint.

As for the suboptimal algorithm, we have the following upper bound: 


$$
B E R_{k, n} \leq Q\left(\sqrt{\frac{2 p_{n} \min _{m}\left|\left[\mathbf{R}_{k, n}\right]_{m, m}\right|^{2}}{M \sigma^{2}}}\right) .
$$

Based on the upper bound in (28), we get the following suboptimal subcarrier allocation:

$$
\rho_{k_{n}^{*}, n}=1, \rho_{k^{\prime}, n}=0, \forall k^{\prime} \neq k_{n}^{*}, \forall n
$$

where

$$
k_{n}^{*}=\arg \max _{k}\left\{\min _{m}\left|\left[\mathbf{R}_{k, n}\right]_{m, m}\right|\right\}, \forall n .
$$

Utilizing the algorithm described in Appendix, we obtain the following suboptimal power allocation

$$
p_{n}=\frac{P_{T} * \zeta_{n}}{1+\zeta_{n}^{2}}\left(\sum_{j=1}^{N} \frac{\zeta_{j}}{1+\zeta_{j}^{2}}\right)^{-1}, \forall n,
$$

where $\zeta_{n}=2 P_{T} /\left(M \sigma^{2}\right) \min _{m} \tilde{\gamma}_{k_{n}^{*}, n, m}$.

\section{Complexity and Diversity Analysis}

\subsection{Complexity analysis}

As mentioned earlier, to find the optimal solutions to the NP-hard problem (3), the optimal subcarrier and the optimal power allocation cannot be performed separately. Finding the optimal subcarrier allocation requires $K^{N}$ searches, which has an exponential-time complexity. Furthermore, a power allocation procedure is required for each possibility of the subcarrier allocation.

The proposed suboptimal algorithms effectively reduce the subcarrier allocation complexity, and the complexity is approximated to $\mathcal{O}(K N)$. While $K^{N}$ times power allocation procedures are required for the exhaustive search, the suboptimal methods only need one power allocation procedure. This is to find out $N$ power variables for $N$ subcarriers. The complexity of the proposed suboptimal algorithms consist of two parts, namely 1) subcarrier allocation of $\mathcal{O}(K N)$ and 2) power allocation of $\mathcal{O}(N)$. Hence, the complexity of the suboptimal methods is approximately $K^{N}$ times less than that of the optimal one, because the power allocation is only executed once. Moreover, while the optimal solution to the power allocation must be obtained by numerical methods, the suboptimal method has a closed-form solution for each type of detectors.

\subsection{Diversity analysis}

In this section, we study the diversity gain of the proposed suboptimal algorithms with various detectors, e.g., the ML, ZF, and ZF-SIC detectors, discussed in Section 3. We derive lower bounds on the diversity gain of the proposed algorithms. Using the upper bound on the BER, we can see the impact of multiple receive antennas and multiple users on the diversity order.

For simplicity, we assume that the elements of the channel matrix $\mathbf{H}_{k, n}$ are independent 
zero-mean CSCG random variables with variance $\sigma_{h}^{2}$. As in Appendix, we define $\kappa=P_{T} / \sigma^{2}$ which is proportional to the average SNR.

For the ML detector, when $\kappa \rightarrow \infty$ (i.e., at high SNR), we have the following asymptotic formula for the suboptimal power allocation:

$$
\lim _{\kappa \rightarrow \infty} p_{n}=\frac{P_{T}}{\max _{k} \min _{\mathbf{d} \in \mathcal{D}}\left\|\mathbf{H}_{k, n} \mathbf{d}\right\|^{2}}\left(\sum_{j=1}^{N} \frac{1}{\max _{k} \min _{\mathbf{d} \in \mathcal{D}}\left\|\mathbf{H}_{k, j} \mathbf{d}\right\|^{2}}\right)^{-1}
$$

Thus, using the upper bound in (12), we have

$$
\lim _{\kappa \rightarrow \infty} B E R_{n} \leq\left(2^{M}-1\right) Q\left(\sqrt{\frac{P_{T}}{2 M \sigma^{2}}\left(\sum_{j=1}^{N} \frac{1}{\max _{k} \min _{\mathbf{d} \in \mathcal{D}}\left\|\mathbf{H}_{k, j} \mathbf{d}\right\|^{2}}\right)^{-1}}\right) .
$$

Then, we can show that

$$
\begin{aligned}
\lim _{\kappa \rightarrow \infty} B E R & \leq\left(2^{M}-1\right) Q\left(\sqrt{\frac{P_{T}}{2 M \sigma^{2}}\left(\sum_{n=1}^{N} \frac{1}{\max _{k} \min _{\mathbf{d} \in \mathcal{D}}\left\|\mathbf{H}_{k, n} \mathbf{d}\right\|^{2}}\right)^{-1}}\right) \\
& \leq \sum_{\mathbf{d} \in \mathcal{D}}\left(2^{M}-1\right) Q\left(\sqrt{\frac{P_{T}}{2 M \sigma^{2}} \frac{\min _{n} \max _{k}\left\|\mathbf{H}_{k, n} \mathbf{d}\right\|^{2}}{N}}\right) .
\end{aligned}
$$

Let $\mathbf{w}_{k, n}=\mathbf{H}_{k, n} \mathbf{d}$. Then, $\mathbf{w}_{k, n}$ is a CSCG random vector and $\mathbb{E}\left[\mathbf{w}_{k, n} \mathbf{w}_{k, n}^{\mathrm{H}}\right]=\sigma_{h}^{2}\|\mathbf{d}\|^{2} \mathbf{I}_{Q}$. From this, we can show that $X_{k, n}=\left\|\mathbf{w}_{k, n}\right\|^{2}$ is a chi-square random variable with $2 Q$ degrees of freedom and its probability density function (pdf) becomes

$$
f_{X}(x)=\frac{1}{\left(\sigma_{h}^{2}\|\mathbf{d}\|^{2}\right)^{Q}(Q-1) !} x^{Q-1} \exp \left(\frac{-x}{\sigma_{h}^{2}\|\mathbf{d}\|^{2}}\right) .
$$

The cumulative distribution function (cdf) is

$$
F_{X}(x)=1-\exp \left(\frac{-x}{\sigma_{h}^{2}\|\mathbf{d}\|^{2}}\right) \sum_{q=0}^{Q-1} \frac{1}{q !}\left(\frac{x}{\sigma_{h}^{2}\|\mathbf{d}\|^{2}}\right)^{q} .
$$

As the $\mathbf{w}_{k, n}$ 's are independent, the cdf and pdf of $V_{n}=\max \left\{X_{1, n}, X_{2, n}, \ldots, X_{K, n}\right\}$ are given by

$$
F_{V_{n}}\left(v_{n}\right)=F_{X}^{K}\left(v_{n}\right), \quad f_{V_{n}}\left(v_{n}\right)=K F_{X}^{K-1}\left(v_{n}\right) f_{X}\left(v_{n}\right) .
$$

Then, the pdf of $V=\min \left\{V_{1}, V_{2}, \ldots, V_{N}\right\}$ is 


$$
\begin{aligned}
f_{V}(v) & =N\left[1-F_{V_{n}}(v)\right]^{N-1} f_{V_{n}}(v) \\
& =N K\left[1-F_{X}^{K}(v)\right]^{N-1} F_{X}^{K-1}(v) f_{X}(v) \\
& =c_{1} v^{K Q-1}+o\left(v^{K Q-1+\epsilon}\right),
\end{aligned}
$$

where $c_{1}>0$ is a constant and $\epsilon>0$. Thus, according to [24], we can show that

$$
\begin{aligned}
\mathbb{E}[B E R] & \leq \sum_{\mathbf{d} \in \mathcal{D}}\left(2^{M}-1\right) \mathbb{E}\left[Q\left(\sqrt{\frac{P_{T}}{2 M \sigma^{2}} \frac{\min _{n} \max _{k}\left\|\mathbf{H}_{k, n} \mathbf{d}\right\|^{2}}{N}}\right)\right] \\
& \leq \max _{\mathbf{d} \in \mathcal{D}} c_{2} \mathbb{E}\left[Q\left(\sqrt{\frac{P_{T}}{2 M \sigma^{2}} \frac{\min _{n} \max _{k}\left\|\mathbf{H}_{k, n} \mathbf{d}\right\|^{2}}{N}}\right)\right] \\
& =c_{3} \gamma_{\mathbf{d}}^{-K Q}+o\left(\gamma_{\mathbf{d}}^{-(K Q+1)}\right),
\end{aligned}
$$

where $\gamma_{\mathbf{d}}=\sigma_{h}^{2}\|\mathbf{d}\|^{2} P_{T} / \sigma^{2}$ and $c_{2}, c_{3}>0$ are constants. This shows that the diversity gain is $K Q$, i.e., the $\mathrm{ML}$ detector can achieve a full multiuser and receive diversity with the proposed suboptimal algorithm.

When M_eig algorithm is exploited for the ZF detector, we have

$$
\begin{aligned}
\lim _{\kappa \rightarrow \infty} B E R & \leq \frac{1}{N} \sum_{n=1}^{N} Q\left(\sqrt{\frac{2 P_{T}}{M \sigma^{2}}\left(\sum_{n=1}^{N} \frac{1}{\max _{k} \lambda_{\min }\left(\mathbf{H}_{k, n}^{\mathrm{H}} \mathbf{H}_{k, n}\right)}\right)^{-1}}\right) \\
& \leq Q\left(\sqrt{\frac{2 P_{T}}{M \sigma^{2}} \frac{\min _{n} \max _{k} \lambda_{\min }\left(\mathbf{H}_{k, n}^{\mathrm{H}} \mathbf{H}_{k, n}\right)}{N}}\right) .
\end{aligned}
$$

Let $X_{k, n}=\lambda_{\text {min }}\left(\mathbf{H}_{k, n}^{\mathrm{H}} \mathbf{H}_{k, n}\right) / \sigma_{h}^{2}$. When $Q=M$, the pdf of the smallest eigenvalue is given by [25] $f_{X}(x)=M \exp (-M x)$, and the cdf is $F_{X}(x)=1-\exp (-M x)$. Let $V_{n}=\max \left\{X_{1, n}, X_{2, n}, \ldots, X_{K, n}\right\}$ and $V=\min \left\{V_{1}, V_{2}, \ldots, V_{N}\right\}$, then the pdf of $V$ is

$$
\begin{aligned}
f_{V}(v) & =N K M\left\{1-[1-\exp (-M v)]^{K}\right\}^{N-1} \times[1-\exp (-M v)]^{K-1} \exp (-M v) \\
& =c_{4} v^{K-1}+o\left(v^{K-1+\epsilon}\right),
\end{aligned}
$$

where $C_{4}>0$ is a constant. Then according to [24], we have that

$$
\mathbb{E}[B E R] \leq c_{5} \gamma_{\Delta}^{-K}+o\left(\gamma_{\Delta}^{-(K+1)}\right),
$$

where $\gamma_{\Delta}=\sigma_{h}^{2} P_{T} / \sigma^{2}$ and $c_{5}>0$ is a constant. If $Q>M$, we can show that the diversity order becomes $K(Q-M+1)$ using the result in [25]. This shows that a full multiuser diversity gain (but not a full receive diversity gain) can by achieved by the ZF detector with M_eig algorithm.

For SIC, when the suboptimal algorithm is used to allocate subcarrier and power, we show that 


$$
\begin{aligned}
\lim _{\kappa \rightarrow \infty} B E R & \leq \frac{1}{N} \sum_{n=1}^{N} Q\left(\sqrt{\frac{2 P_{T}}{\sigma^{2} M}\left(\sum_{n=1}^{N} \frac{1}{\max _{k} \min _{m}\left|\left[R_{k, n}\right]_{m, m}\right|^{2}}\right)^{-1}}\right) \\
& \leq Q\left(\sqrt{\frac{2 P_{T}}{\sigma^{2} M N} \min _{n} \max _{k} \min _{m}\left|\left[R_{k, n}\right]_{m, m}\right|^{2}}\right) .
\end{aligned}
$$

According to [26], $X_{k, n, m}=\left|\left[R_{k, n}\right]_{m, m}\right|^{2}$ follows a chi-square distribution with $2(M-m+1)$ degrees of freedom given as

$$
\begin{aligned}
& f_{X_{k, n, m}}(x)=\frac{1}{\left(\sigma_{h}^{2}\right)^{M-m+1}(M-m) !} x^{M-m} \exp \left(-x / \sigma_{h}^{2}\right), \\
& F_{X_{k, n, m}}(x)=1-\exp \left(-x / \sigma_{h}^{2}\right) \sum_{i=0}^{M-m} \frac{1}{i !}\left(x / \sigma_{h}^{2}\right)^{i} .
\end{aligned}
$$

The cdf and pdf of $Y_{k, n}=\min \left\{X_{k, n, 1}, X_{k, n, 2}, \ldots, X_{k, n, M}\right\}$, respectively, are given by

$$
F_{Y}(y)=1-\prod_{m=1}^{M}\left[1-F_{X_{k, n, m}}(y)\right], \quad f_{Y}(y)=\sum_{i=1}^{M} f_{X_{k, n, i}}(y) \prod_{m=1, m \neq i}^{M}\left[1-F_{X_{k, n, m}}(y)\right] .
$$

Let $V_{n}=\max \left\{Y_{1, n}, Y_{2, n}, \ldots, Y_{K, n}\right\}$ and $V=\min \left\{V_{1}, V_{2}, \ldots, V_{N}\right\}$. Then, the pdf of $V$ is

$$
f_{V}(v)=N K\left[1-F_{Y}^{K}(v)\right]^{N-1} F_{Y}^{K-1}(v) f_{Y}(v)=c_{6} V^{K-1}+o\left(v^{K-1+\epsilon}\right),
$$

where $c_{6}>0$ is a constant. Then according to [24], we have that

$$
\mathbb{E}[B E R] \leq c_{7} \gamma_{\Delta}^{-K}+o\left(\gamma_{\Delta}^{-(K+1)}\right),
$$

where $\gamma_{\Delta}=\sigma_{h}^{2} P_{T} / \sigma^{2}$ and $c_{7}>0$ is a constant. This shows that the diversity order with the SIC detector is $K$.

\section{Simulation Results}

In this section, the performances of the proposed algorithms are evaluated. For the purpose of comparison, we also simulate the performance of two conventional algorithms which will be referred to as Opt_u and Prod_opt in the following discussion. Opt_u algorithm is to allocate power uniformly, and then use the mean BER to choose the optimal subcarrier allocation. With Pro_opt algorithm, each subcarrier is allocated to the user with the highest eigenvalue product [27] which is to maximize the capacity, and then the power is allocated with the optimal approach instead of the "water-filling" solution. Due to the high computational complexity of the optimal algorithms due to the exhaustive search, we first compare the BER performances of the proposed suboptimal algorithm and the optimal algorithm in a small-scale system with $N=8$ subcarriers and $K=4$ users. Then, we compare the proposed suboptimal algorithm and the two conventional algorithms in a large system with one BS that serves $K=8$ or 16 users sharing $N=64$ subcarriers. For simplicity, we assume the time-domain channel matrices $\mathbf{G}_{k}(l), l \in \mathcal{L}$ are independent random matrices, each having entries independently distributed as $\mathcal{C N}(0,1 / L)$. The detailed simulation parameters are listed in Table 1. 
Table 1. Simulation parameters

\begin{tabular}{|l|l|l|}
\hline Parameter & Description & Value \\
\hline $\mathrm{M}$ & Number of antennas at BS & 2 \\
\hline $\mathrm{Q}$ & Number of antennas at each user & 2 \\
\hline $\mathrm{L}$ & Number of multipath & 4 \\
\hline $\mathrm{N}$ & Number of subcarriers & $\{8\}\{64\}$ \\
\hline $\mathrm{K}$ & Number of users & $\{4\}\{8,16\}$ \\
\hline $\mathrm{G}_{k}(l)$ & Time-domain channel matrices & i.i.d with distribution $\mathcal{C N}\left(0, \mathbf{I}_{Q}\right)$ \\
\hline
\end{tabular}

Fig. 2 shows the BER of the ML detector. The BER performance yielded by the proposed suboptimal is close to that of the optimal algorithm, as shown in Fig. 2. (a), especially in the high SNR region. As expected, the proposed suboptimal algorithm always performs better than Prod_opt and Opt_u algorithms. For example, at a BER of $10^{-6}$, the suboptimal algorithm has a gain of about $1 \mathrm{~dB}$ as compared to Opt_u and Prod_opt algorithms when $N=64$ and $K=16$. This is because the proposed suboptimal algorithm allocates subcarrier and power to minimize the BER of the system, while Prod_opt algorithm does not concern the actually employed MIMO detectors and Opt_u algorithm only assumes equal power loading over subcarriers.

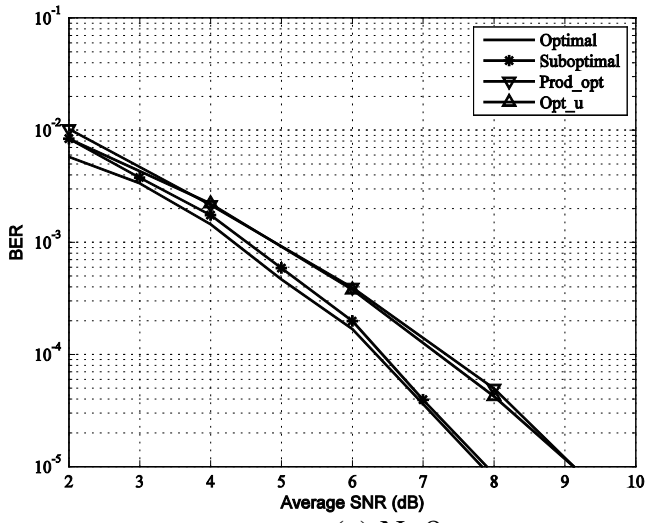

(a) $\mathrm{N}=8$

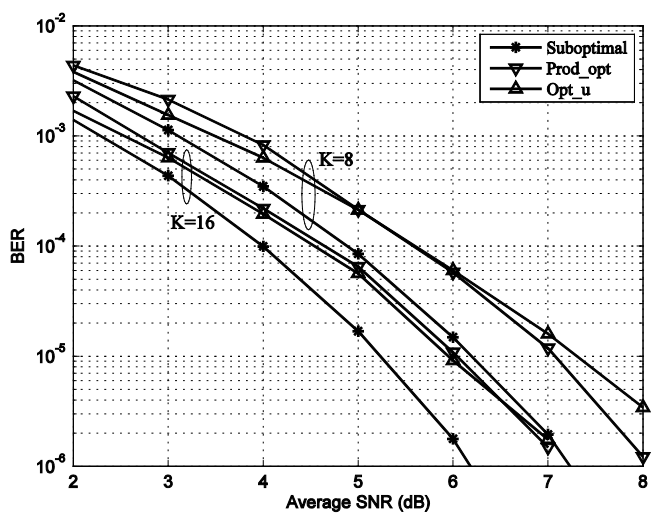

(b) $\mathrm{N}=64$

Fig. 2. Average BER performance for the MIMO-OFDM system with the ML detector

In Fig. 3, we present the BER results for the ZF detector with different resource allocation algorithms. It is observed that M_eig and M_diag algorithms appear to be quite close to the optimal algorithm from Fig. 3. (a). We can also see that M_diag algorithm always outperforms M_eig algorithms. This result is expected for the reason that the upper bound in (24) is tighter than the upper bound in (21). From Fig. 3. (b), M_diag algorithm has a gain of about $0.7 \mathrm{~dB}$ as compared to Prod_opt algorithm and about $1.6 \mathrm{~dB}$ as compared to Opt_u algorithm at a BER of $10^{-6}$ when there are $K=16$ users. 


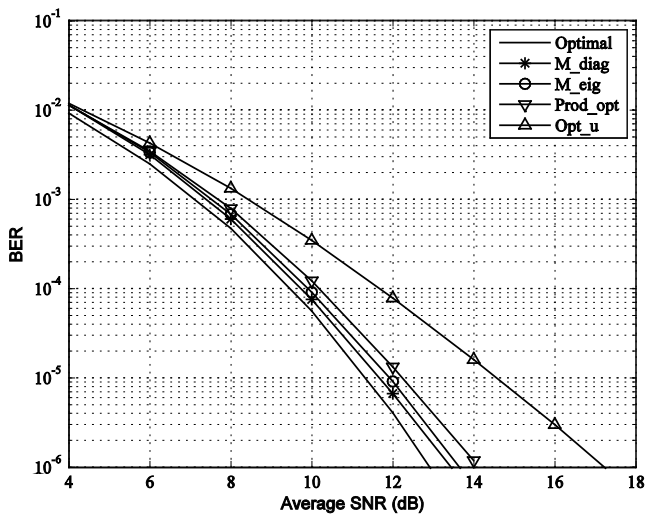

(a) $\mathrm{N}=8$

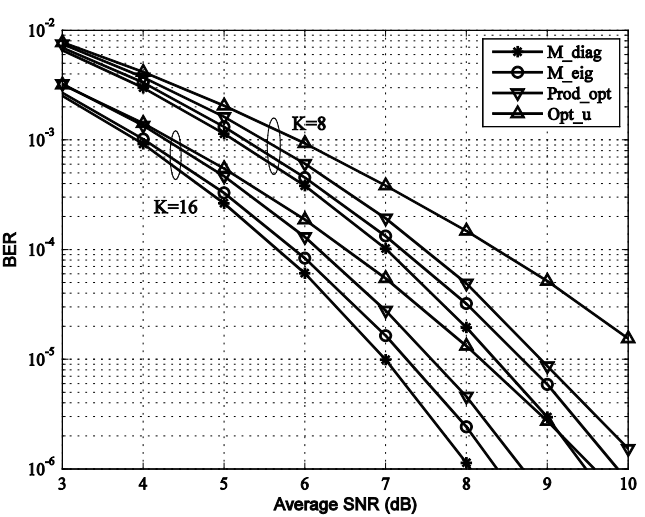

(b) $\mathrm{N}=64$

Fig. 3. Average BER performance for the MIMO-OFDM system with the ZF detector

Fig. 4 demonstrates the BER performance versus average SNR for the ZF-SIC detector. In Fig. 4. (a), we see that the proposed suboptimal has nearly the same BER performance as the optimal algorithm. We examine that the proposed suboptimal algorithm has lower BER than Pro_opt and Opt_u algorithms. For example, at $S N R=8 \mathrm{~dB}$, the suboptimal algorithm outperforms Opt_u algorithm by about one order of magnitude when there are $K=8$ users. At a BER of $10^{-6}$, the SNR gap between the proposed suboptimal algorithm and Prod_opt algorithm is about $0.8 \mathrm{~dB}$.

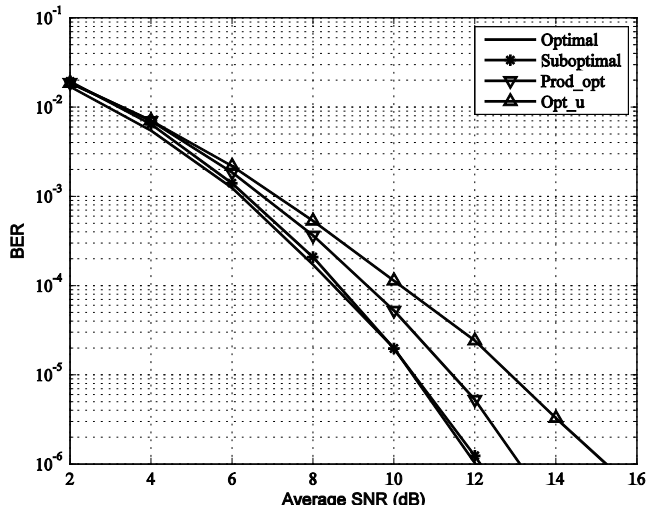

(a) $\mathrm{N}=8$

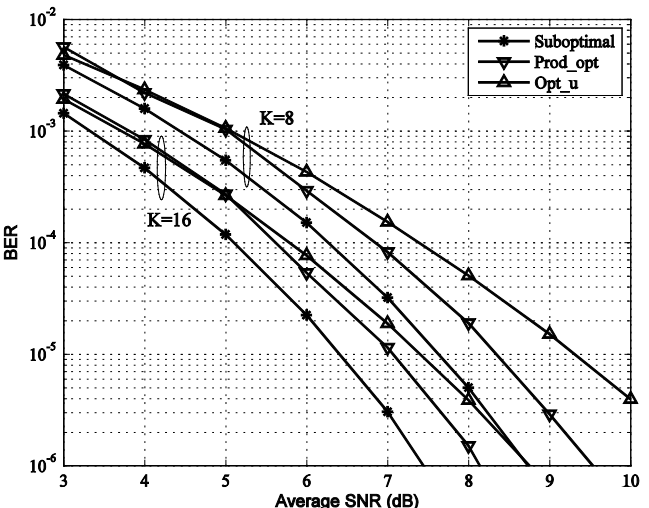

(b) $\mathrm{N}=64$

Fig. 4. Average BER performance for the MIMO-OFDM system with the ZF-SIC detector

It is noted that the BER decreases with the number of users, which can be explained by the multiuser diversity effect. As the number of users increases, the probability that a user of high SNR is assigned to a given subcarrier increases as well, resulting in more efficient use of power and better performance. Moreover, simulation results verified that capacity-oriented resource allocation algorithms can not achieve a good performance when a realistic MIMO detection method is employed. In contrast, the proposed suboptimal algorithm for a specified detector yields an improve BER performance with a much lower complexity. 


\section{Conclusion}

This paper investigated the BER-optimized resource allocation for multiuser MIMO-OFDM systems with considering that realistic MIMO detection techniques are employed. As the BER performance is depending on the actually employed MIMO detectors, we proposed different subcarrier and power allocation algorithms for three types of conventional MIMO detectors, including the ML detector, linear detectors and SIC detectors. By separating the subcarrier and power allocation, a low-complexity subcarrier and close-form power allocation algorithm is derived for each detector. We also analyzed the diversity gain of the suboptimal algorithms. It is shown that the ML, ZF and SIC detectors can exploit the full multiuser diversity with the proposed low-complexity suboptimal resource allocation algorithms. The simulation results show that, for each detector, our proposed suboptimal algorithm can achieve a comparable performance with the optimal algorithm and outperforms conventional algorithms in terms of BER.

\section{Appendix}

With the subcarrier allocation $\left\{\rho_{k, n}\right\}$ is determined, Problem (6) should be solved to allocate power. In this section, a suboptimal power allocation is obtained by replacing the $B E R_{k_{n}^{*}, n}$ with the upper bounds on the BER. The average SNR can be defined as $P_{T} /\left(N M \sigma^{2}\right)$. Then $\kappa=P_{T} / \sigma^{2}$ is proportional to the average SNR. Let $\mu_{n}=p_{n} / P_{T}$ denotes the power partition coefficient and $\beta_{n}$ denotes the rest parameters of the upper bound on $B E R_{k_{n}^{*}, n}$. More specifically, for the ML detector, $\beta_{n}=\min _{\mathbf{d} \in \mathcal{D}}\left\|\mathbf{H}_{k_{n}^{*}, n} \mathbf{d}\right\|^{2} /(2 M)$ from the upper bound in (12); for the ZF detector, $\beta_{n}=2 \lambda_{\min }\left(\mathbf{H}_{k_{n}^{*}, n}^{\mathrm{H}} \mathbf{H}_{k_{n}^{*}, n}\right) / M$ from the upper bound in (21) and $\beta_{n}=2 /\left(M \max _{m}\left[\left(\mathbf{H}_{k_{n}^{*}, n}^{\mathrm{H}} \mathbf{H}_{k_{n}^{*}, n}\right)^{-1}\right]_{m, m}\right)$ from the upper bound in (24); for the ZF-SIC detector, $\beta_{n}=2 \min _{m}\left|\left[\mathbf{R}_{k_{n}^{*}, n}\right]_{m, m}\right|^{2} / M$ from the upper bound in (28). Then Problem (6) can be transformed into the following problem:

$$
\begin{aligned}
& \min \sum_{n=1}^{N} Q\left(\sqrt{\kappa \mu_{n} \beta_{n}}\right) \\
& \text { s.t. } \sum_{n=1}^{N} \mu_{n}=1 .
\end{aligned}
$$

The $\mu_{n}$ can be obtained by means of the Lagrange method, i.e., by solving the following system of equations:

where

$$
\frac{\partial L}{\partial \mu_{n}}=0, \forall n \in \mathcal{N}
$$

$$
L=\sum_{n=1}^{N} Q\left(\sqrt{\kappa \mu_{n} \beta_{n}}\right)+\lambda\left(\sum_{n=1}^{N} \mu_{n}-1\right)
$$


is the Lagrangian function of the optimal problem (40) and $\lambda$ is the Lagrangian multiplier. We obtain

$$
\lambda=\frac{\sqrt{\kappa \beta_{n}}}{2 \sqrt{2 \pi \mu_{n}}} \exp \left(-\frac{\kappa \mu_{n} \beta_{n}}{2}\right), \forall n \in \mathcal{N} \text {. }
$$

From (43), we have

$$
\frac{\mu_{n}}{\mu_{j}}=\frac{\beta_{n} \exp \left(-\kappa \mu_{n} \beta_{n}\right)}{\beta_{j} \exp \left(-\kappa \mu_{j} \beta_{j}\right)} .
$$

From the constraint in (40), we obtain

$$
\mu_{n}=\frac{\beta_{n} \exp \left(-\kappa \mu_{n} \beta_{n}\right)}{\sum_{j=1}^{N} \beta_{j} \exp \left(-\kappa \mu_{j} \beta_{j}\right)}
$$

Consider the behavior of $\mu_{n}$ in two opposite situations, when $\kappa \rightarrow 0$ (i.e., at low SNR) and $\kappa \rightarrow \infty$ (i.e., at high SNR). For the fist case, $\exp \left(-\kappa \mu_{n} \beta_{n}\right)$ approaches 1 . We obtain

$$
\lim _{\kappa \rightarrow 0} \mu_{n}=\frac{\beta_{n}}{\sum_{j=1}^{N} \beta_{j}}
$$

In the second case, let us consider the Laurent expansions of (45),

$$
\mu_{n}=\mu_{n_{0}}+\mu_{n_{1}} \kappa^{-1}+\mu_{n_{2}} \kappa^{-2}+\cdots
$$

where $\mu_{n_{i}}$ 's are the expansion coefficients. Substituting (47) into (45), we obtain

$$
\mu_{n_{0}}+\mu_{n_{1}} \kappa^{-1}+\mu_{n_{2}} \kappa^{-2}+\cdots=\frac{\beta_{n} \exp \left(-\beta_{n} \mu_{n_{0}} \kappa-\beta_{n} \mu_{n_{1}}-\beta_{n} \mu_{n_{2}} \kappa^{-1}-\cdots\right)}{\sum_{j=1}^{N} \beta_{j} \exp \left(-\beta_{j} \mu_{j_{0}} \kappa-\beta_{j} \mu_{j_{1}}-\beta_{n} \mu_{j_{2}} \kappa^{-1}-\cdots\right)}
$$

Taking into account that in (48) $\kappa^{-i} \rightarrow 0$ for $\forall i \geq 1$ when $\kappa \rightarrow \infty$ and multiplying the numerator and denominator of the right-hand side of (48) by $\exp \left(\beta_{n} \mu_{n_{0}} \kappa\right)$, we obtain

$$
\mu_{n_{0}}=\frac{\beta_{n} \exp \left(-\beta_{n} \mu_{n_{1}}\right)}{\sum_{j=1}^{N} \beta_{j} \exp \left[-\left(\beta_{j} \mu_{j_{0}}-\beta_{n} \mu_{n_{0}}\right) \kappa-\beta_{j} \mu_{j_{1}}\right]} .
$$

Since $\mu_{n_{0}}$ in the left-hand side of (49) and the numerator of the right-hand side of (49) do not depend on $\kappa$, the denominator also does not depend on $\kappa$. Therefore, we have

$$
\beta_{n} \mu_{n_{0}}=\beta_{j} \mu_{j_{0}} .
$$

Considering the constraint in (40), we obtain

$$
\lim _{\kappa \rightarrow \infty} \mu_{n}=\mu_{n_{0}}=\frac{1}{\beta_{n}}\left(\sum_{j=1}^{N} \frac{1}{\beta_{j}}\right)^{-1} .
$$

In order to simultaneously satisfy (46) and (51), we can find an approximation of $\mu_{n}$, which is expressed as follows 


$$
\mu_{n}=\frac{\zeta_{n}}{1+\zeta_{n}^{2}}\left(\sum_{j=1}^{N} \frac{\zeta_{j}}{1+\zeta_{j}^{2}}\right)^{-1}, \forall n \in \mathcal{N},
$$

where $\zeta_{n}=\beta_{n} \kappa$.Thus, we have

$$
p_{n}=\frac{P_{T} \zeta_{n}}{1+\zeta_{n}^{2}}\left(\sum_{j=1}^{N} \frac{\zeta_{j}}{1+\zeta_{j}^{2}}\right)^{-1}, \forall n \in \mathcal{N} .
$$

\section{References}

[1] "Ieee approved draft standard for it - telecommunications and information exchange between systems - LAN/MAN - specific requirements - part 11: Wireless LAN medium access control and physical layer specifications- amd 4: Enhancements for very high throughput for operation in bands below 6ghz,” IEEE P802.11ac/D7.0, pp. 1-456, December, 2013. Article (CrossRef Link)

[2] "IEEE standard for local and metropolitan area networks part 16: Air interface for fixed and mobile broadband wireless access systems amendment 2: Physical and medium access control layers for combined fixed and mobile operation in licensed bands and corrigendum 1,” IEEE Std 802.16e-2005 and IEEE Std 802.16-2004/Cor 1-2005 (Amendment and Corrigendum to IEEE Std 802.16-2004), pp. 1-822, 2006. Article (CrossRef Link)

[3] C. E. Huang and C. Leung, "BitQoS-aware resource allocation for multi-user mixed-traffic OFDM systems," IEEE Transactions on Vehicular Technology, vol. 61, no. 5, pp. 2067-2082, June, 2012. Article (CrossRef Link)

[4] G. Femenias and F. R.-Palou, "Scheduling and Resource Allocation in Downlink Multiuser MIMO-OFDMA Systems," IEEE Transactions on Communications, vol. 64, no. 5, pp. 2019-2034, May, 2016. Article (CrossRef Link)

[5] N. A. Odhah, E.S. Hassan and et al., "Adaptive Resource Allocation Algorithms for Multi-user MIMO-OFDM Systems," Wireless Personal Communications, vol. 80, no. 1, pp. 51-69, September, 2015. Article (CrossRef Link)

[6] M. A. Ali, S. A-Ghadhban and A. S. H. Mahnound, "Resource allocation scheme for MIMO-OFDMA systems with proportional fairness constraints," in Proc. of 2012 IEEE 8th international conference on wireless and Mobile Computing, Networking and Communication (WiMob), pp. 512-516, October 8-10, 2012. Article (CrossRef Link)

[7] W.-C. Pao, W.-T. Lou, Y.-F. Chen, and D.-C.Chang, "Resource allocation for multiple input multiple output-orthogonal frequency division multiplexing-based space division multiple access systems,” IET Communications, vol. 8, no. 18, pp. 3424-3434, December, 2014. Article (CrossRef Link)

[8] M. G. Adian and H. Aghaeinia, "Resource allocation in MIMO-OFDM -based cooperative cognitive radio networks,” IEEE Transactions on Communications, vol. 62, no. 7, pp. 2224-2235, July, 2014. Article (CrossRef Link)

[9] Z. Hu, G. Zhu, Y. Xia and G. Liu, "Multiuser subcarrier and bit allocation for MIMO-OFDM systems with perfect and partial channel information,” in Proc. of 2004 Wireless Communications and Networking Conference, vol. 2, pp. 1188-1193, March 21-25, 2004. Article (CrossRef Link)

[10] W. L. Ho and Y.-C. Liang, "Optimal resource allocation for multiuser MIMO-OFDM systems with user rate constraints," IEEE Transactions on Vehicular Technology, vol. 58, no. 3, pp. 1190-1203, March, 2009. Article (CrossRef Link)

[11] C. Hsu, P. L. Yeoh and B. S. Krongold, "Power minimization in cooperative MIMO-OFDM systems with user fairness constraints,” in Proc. of 2015 IEEE Wireless Communications and Networking Conference (WCNC), pp. 93-98, March 9-12, 2015. Article (CrossRef Link)

[12] M. Moretti, L. Sanguinetti and X. Wang, "Resource allocation for power minimization in the downlink of thp-based spatial multiplexing MIMO-OFDM systems," IEEE Transactions on Vehicular Technology, vol. 64, no. 1, pp. 405-411, April, 2015. Article (CrossRef Link) 
[13] L. Goldfeld, V. Lyandres and D. Wulich, "Minimum ber power loading for OFDM in fading channel," IEEE Transactions on Communications, vol. 50, no. 11, pp. 1729-1733, November, 2002. Article (CrossRef Link)

[14] N. Wang and S. D. Blostein, “Approximate minimum BER power allocation for MIMO spatial multiplexing systems,” IEEE Transactions on Communications, vol. 55, no. 1, pp. 180-187, January, 2007. Article (CrossRef Link)

[15] D. Qin and Y. Wang, "BER Minimization for AF Relay-Assisted OFDM Systems," IEEE Communications Letters, vol. 19, no. 3, pp. 495-498, March, 2015. Article (CrossRef Link)

[16] A. S. Bedi, J. Akhtar, K. Rajawat and A. K. Jagannatham, "BER-Optimized Precoders for OFDM Systems With Insufficient Cyclic Prefix," IEEE Communications Letters, vol. 20, no. 2, pp. 280-283, February, 2016. Article (CrossRef Link)

[17] V. Lau, Y. Liu, and T.-A. Chen, "On the design of MIMO block fading channels with feedback-link capacity constraint,” IEEE Transactions on Communications, vol. 52, no. 1, pp. 62-70, January, 2004. Article (CrossRef Link)

[18] Q. H. Spencer, A. L. Swindlehurst and M. Haardt, "Zero-forcing methods for downlink spatial multiplexing in multiuser MIMO channels,” IEEE Transactions on Signal Processing, vol. 52, no. 2, pp. 461-471, February, 2004. Article (CrossRef Link)

[19] D. Tse, Fundamentals of wireless communication, Cambridge university press, United Kingdom, 2005. Article (CrossRef Link)

[20] J. Choi, Optimal combining and detection: statistical signal processing for communications, Cambridge University Press, United Kingdom, 2010. Article (CrossRef Link)

[21] M. A. Juang and M. B. Pursley, "Power loading for OFDM in tactical packet radio systems," in Proc. of 2011 Military Communication Conference, pp. 572-577, November 7-10, 2011. Article (CrossRef Link)

[22] S. Verdu, Multiuser detection, Cambridge university press, United Kingdom, 1998.

[23] R.A. Horn, and C.R Johnson, Matrix analysis, Cambridge University Press, England, 2012. Article (CrossRef Link)

[24] Z. Wang and G. B. Giannakis, “A simple and general parameterization quantifying performance in fading channels,” IEEE Transactions on Communications, vol. 51, no. 8, pp. 1389-1398, August, 2003. Article (CrossRef Link)

[25] A. Edelman, "Eigenvalues and condition numbers of random matrices," SIAM Journal on Matrix Analysis and Applications, vol. 9, no. 4, pp. 543-560, October, 1988. Article (CrossRef Link)

[26] H. Kim and H. Park, "Iterative interference cancellation algorithms for the v-blast system," in Proc. of 2005 IEEE 16th International Symposium on Personal, Indoor and Mobile Radio, vol. 4, pp. 2435-2440, September 11-14, 2005. Article (CrossRef Link)

[27] J. Xu, S.-J. Lee, W.-S. Kang and J.-S. Seo, “Adaptive resource allocation for MIMO-OFDM based wireless multicast systems,” IEEE Transactions on Broadcasting, vol. 56, no. 1, pp. 98-102, March, 2010. Article (CrossRef Link)

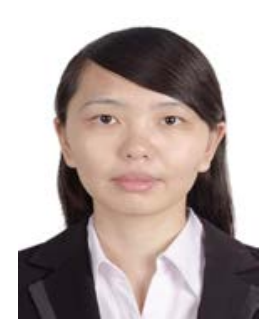

Jing Mao received the B.E. degree in electronics information engineering from Nankai University, Tianjin, China, in 2010. She is currently pursuing the Ph.D. degree in the State Key Laboratory of Advanced Optical Communication Systems and Networks Peking University, Beijing, China. His research interests are in the areas of wireless communications and multiple-input and multiple-output systems. 


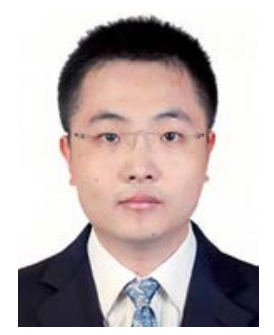

Chen Chen (M'10) received the B.S. and Ph.D. degrees in electronics engineering from Peking University, Beijing, China, in 2004 and 2009, respectively. He is now an Associate Professor in School of Electronics Engineering and Computer Science, Peking University, Beijing. His research interests include wireless communications and networking, network information theory, signal processing of wireless communications, wireless network coding, array/smart antenna, and multiple-input and multiple-output systems.

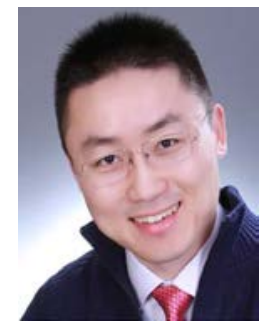

Lin Bai (M’13) received the B.Sc. degree in electronics and information engineering from Huazhong University of Science and Technology, Wuhan, China, in 2004, the M.Sc. (with distinction) degree in communication systems from the University of Wales, Swansea, U.K., in 2007, and the Ph.D. degree in advanced telecommunications from the School of Engineering, Swansea University, U.K., in 2010. Since 2011, he has been with the School of Electronics and Information Engineering, Beihang University (Beijing University of Aeronautics and Astronautics, BUAA), Beijing, China, as an Associate Professor. He is the author of the books, Low Complexity MIMO Detection (Springer, 2012) and Low Complexity MIMO Receivers (Springer, 2014). His research interests include signal processing of wireless communications, particularly multiple-input multiple output systems, array/smart antenna, and lattice-based approaches. Dr. Bai received an IEEE Communications Letters Exemplary Reviewers Certificate for 2012 and the Best Paper Award from ICNS 2013 (Conference). Currently, he is an Editor of the KSII Transactions on Internet and Information Systems and served as a Guest Editor of the International Journal of Distributed Sensor Networks from 2012 to 2014.

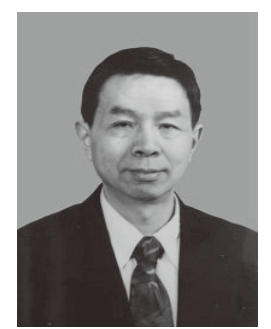

Haige Xiang graduated from department radio-electronics in Peking University in 1964. He began work in Peking University. He is currently a Professor of the School of Electronics Engineering and Computer Science in Peking University. His research fields are digital communication and signal processing, such as wireless and satellite communication networks, multi-user detections and interference cancellation, multiple carrier communications and OFDM systems, smart antenna and MIMO systems, and channel code technique.

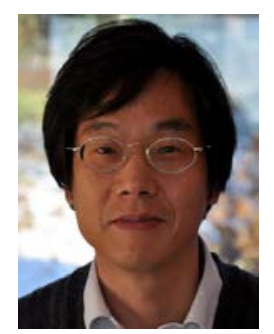

Jinho Choi (M’91-SM’02) was born in Seoul, Korea. He received the B.E. (magna cum laude) degree in electronics engineering from Sogang University, Seoul, in 1989, and the M.S.E. and Ph.D. degrees in electrical engineering from Korea Advanced Institute of Science and Technology (KAIST), Daejeon, in 1991 and 1994, respectively. He is with Gwangju Institute of Science and Technology (GIST) as a Professor. Prior to joining GIST in 2013, he was with the College of Engineering, Swansea University, United Kingdom, as a Professor/Chair in wireless. His research interests include wireless communications and array/statistical signal processing. He has authored two books published by Cambridge University Press in 2006 and 2010. Prof. Choi received the 1999 Best Paper Award for Signal Processing from EURASIP, 2009 Best Paper Award from WPMC (Conference). Since 2015, he has been Associate Editor of IEEE Communications Letters and an Editor of Journal of Communications and Networks. He has also served as an Associate Editor of IEEE Transactions on Vehicular Technology from 2005 to 2007 and ETRI Journal. 